\title{
Design and Optimization of Index-Guiding Photonic Crystal Fiber Gas Sensor
}

\author{
Saeed OLYAEE ${ }^{*}$ and Alieh NARAGHI \\ Nano-photonics and Optoelectronics Research Laboratory (NORLab), Faculty of Electrical and Computer Engineering, \\ Shahid Rajaee Teacher Training University, Tehran, Iran \\ ${ }^{*}$ Corresponding author: Saeed OLYAEE_ E-mail: s_olyaee@srttu.edu
}

\begin{abstract}
An index guiding photonic crystal fiber used in gas sensing applications is presented. The dependency of the confinement loss and relative sensitivity on the fiber parameters and wavelength is numerically investigated by using the full-vectorial finite element method (FEM). The simulations showed that the gas sensing sensitivity increased with an increase in the core diameter and a decrease in the distance between centers of two adjacent holes. Increasing the hole size of two outer cladding rings, this structure simultaneously showed up to $10 \%$ improved sensitivity, and the confinement loss reached $6 \times 10^{-4}$ times less than that of the prior sensor at the wavelength of $1.5 \mu \mathrm{m}$. This proved the ability of this fiber used in gas and chemicals sensing applications.
\end{abstract}

Keywords: Confinement loss, evanescent field, gas sensor, relative sensitivity, photonic crystal fiber

Citation: Saeed OLYAEE and Alieh NARAGHI, "Design and Optimization of Index-Guiding Photonic Crystal Fiber Gas Sensor," Photonic Sensors, vol. 3, no. 2, pp. 131-136, 2013.

\section{Introduction}

In the structure of photonic crystal fibers (PCFs), there are various air-holes which preset the length of fibers. The PCFs, which possess the sole structure and inimitable characteristics, are tremendously utilized in numerous fields of applications such as communication and sensing devices $[1,2]$. In the meantime, the endless single mode, high nonlinearity, high birefringence, and flexible chromatic dispersion are some of unique characteristics of PCFs [3-12].

Air holes, running along the length of the fiber, create new abilities for the appropriate interaction between light and gases or liquids through evanescent fields in the holes. The air-core photonic band gap fiber, which is one of the interesting types of PCFs, magnifies the interaction of the evanescent field with penetration into the holes to the tremendously high; moreover, a huge amount of light in this structure can be confined within the hollow core, which causes an increase in the interaction $[13,14]$.

The narrow spectral band for propagation restricts the applications of the photonic band gap fiber (PBGF); additionally, periodic microstructure dimensions, for having the band gap effect, require the precise and stringent control which is another limitation. However, in order to avoid these limitations, some observational researches used the evanescent field based on the index-guiding PCF [15-17]. But in the index guiding PCF, the interaction of light with gas samples has been limited, and so the sensitivity is low.

Received: 28 August 2012 / Revised version: 12 January 2013

(C) The Author(s) 2013. This article is published with open access at Springerlink.com 
In this paper, an evanescent field gas sensor based on the photonic crystal fiber is introduced, in which the core consists of an air hole with dimensions smaller than the dimensions of the cladding holes to satisfy the effective index guiding criterion. Due to the central hole, the difference between the refractive indices of the core and cladding dropped, more light would penetrate into the cladding, and the interaction between light and gases and thus the sensitivity increased [18]. The larger central hole diameter $d_{c}$ showed the higher evanescent field fraction, nevertheless, the central hole diameter should be less than the cladding hole diameter $d$, to satisfy the effective index guiding criterion. Although in [18] due to the smaller air hole in the center, the evanescent field interaction was enhanced, but this type of PCFs had a huge confinement loss. In this paper, we introduce an optimal structure to reduce the confinement loss by using a 5-ring hole and increasing the hole dimension of two outer cladding rings. We investigated the effect of changing the dimensions of the central hole, $d_{c}$, and the cladding holes, $d$, the center-to-center distance between two adjacent holes, $a$, and the wavelength on the relative sensitivity and confinement loss and introduced the optimal structure for simultaneously achieving more sensitivity and less confinement losses. Figures 1(a) and 1(b) show the PCF structure of [18] and proposed structure, respectively.

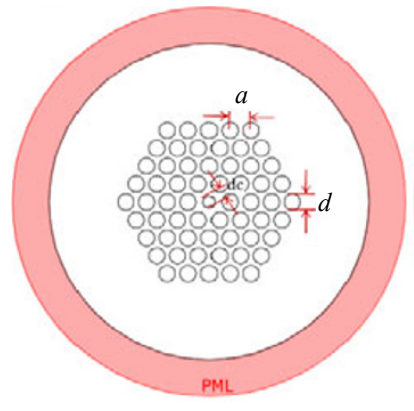

(a)

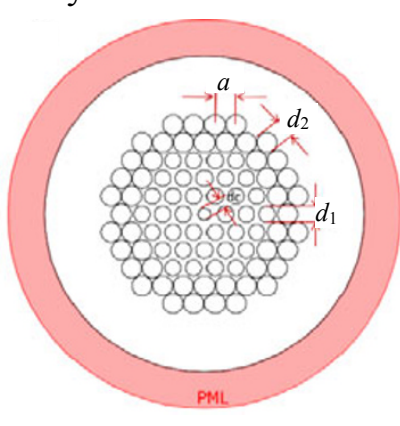

(b)
Fig. 1 Cross-section of (a) prior PCF with a central air-hole defect with the diameter $d_{c}$ [18] and (b) proposed PCF with the same core defect with 5 rings of holes and two outer rings with larger air holes.

\section{Numerical analysis of optical properties}

To review the proposed PCF optical properties, the finite element method (FEM) for solving Maxwell's equations was applied due to its proven reliability and high accuracy for analyzing the PCF $[19,20]$.

One of the parameters in design of the PCF with a finite number of air holes is the leakage loss, which sometimes is known as the confinement loss. Basically, the leakage loss is eliminated when the number of air holes in cladding is infinite. Nevertheless, in the practical structure, the number of air holes is finite; consequently, the modes of such fibers are inherently leaky. And also, we must consider that the imaginary part of its complex propagation constant represents the leakage loss of a mode.

For having an appropriate model of the leakage, an open boundary condition is required, which doesn't create reflection at the boundary. Perfectly matched layers (PMLs) are so far the most efficient absorption boundary condition for this purpose [21, 22]. Here, we use the circular PML as introduced in [23]. The confinement loss $L_{c}$, in decibels per meter is given by

$$
L_{c}=8.686 K_{0} \operatorname{Im}\left[n_{\text {eff }}\right]
$$

where $\operatorname{Im}\left[n_{\text {eff }}\right]$ is the imaginary part of the effective index [23].

If the index difference between the core and cladding can be weakened by the central air hole, a significant confinement loss appears. In prior PCFs, a reduction in the confinement loss has been attempted by increasing the number of hole layers in the cladding [24, 25]. However, imbedding additional air hole layers into PCFs requires highly elaborated processes in the practical manufacture, especially to keep the hole uniformity along both the lateral and axial directions. By changing the dimensions of the cladding air holes, we conclude that varying the diameter of the holes located in inner rings has no significant effect on the 
confinement loss. But by changing the diameter of the outer ring of holes, loss greatly varies.

The evanescent field in the air holes is absorbed by the gas species, and the gas concentration can be obtained from the intensity through the Beer-Lambert law:

$$
I(\lambda)=I_{0}(\lambda) \exp \left[-r \alpha_{m}(\lambda) l C\right]
$$

where $I$ is the output light intensity in the presence of gas, and $I_{0}$ refers the output light intensity without the presence of gas. Also, $\alpha_{m}$, which is a function of the wavelength, is the absorption coefficient of the gas. $l$ and $C$, respectively, denote the length of the PCF used for the gas detection (interaction length) and the gas concentration, and finally, $r$ is a relative sensitivity coefficient defined as

$$
r=\frac{n_{r}}{n_{e}} f
$$

where $n_{r}$ is almost equal to one and refers the refractive index of the gas species. The effective refractive index of the guide mode is presented by $n_{e}$, and $f$ is the fraction of the total power located in the holes; in the meantime, in the typical fiber, $f$ can be calculated by

$$
f=\frac{\int_{\text {holes }}\left(E_{x} H_{y}-E_{y} H_{x}\right) d x d y}{\int_{\text {total }}\left(E_{x} H_{y}-E_{y} H_{x}\right) d x d y} .
$$

The transverse electric and magnetic fields of the mode are introduced by $E_{x}, E_{y}$ and $H_{x}, H_{y}$, respectively. Now, with solving Maxwell's equations by utilizing a finite-element method, the effective refractive index $n_{e}$ and the mode field pattern, $E_{x}, E_{y}$ and $H_{x}, H_{y}$ can be acquired [26].

\section{Results and analysis}

First, we have simulated the structure introduced in [18]. In addition to the sensitivity with the circular PML layer, the confinement loss was calculated at different wavelengths using the FEM method. In all analyses, the cladding refractive index and the holes refractive indices were considered to be equal to 1.5 and 1 , respectively.

For the proposed PCF, in the case of $\alpha=2 \mu \mathrm{m}$ and $2.3 \mu \mathrm{m}$, the hole dimension of two outer cladding rings, $d_{2}$, was considered equal to $1.8 \mu \mathrm{m}$, and in the case of $\alpha=1.8 \mu \mathrm{m}$ and $\alpha=1.6 \mu \mathrm{m}$ we assumed $\quad d_{2}=1.74 \mu \mathrm{m} \quad$ and $\quad d_{2}=1.56 \mu \mathrm{m}$, respectively.

Figures 2 and 3 show the relative sensitivities for the conventional PCF with respect to the wavelength

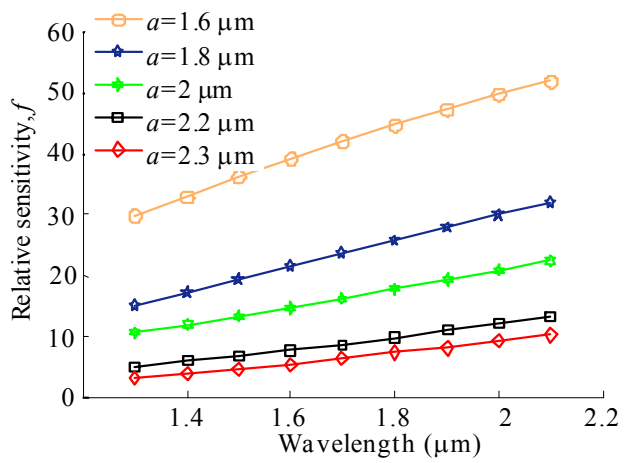

Fig. 2 Relative sensitivity in terms of the wavelength for the prior PCF with $d=1.4 \mu \mathrm{m}$ and $d_{c}=1 \mu \mathrm{m}$.

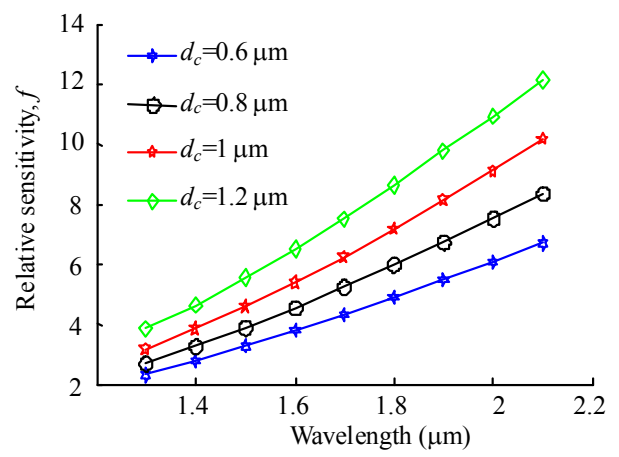

Fig. 3 Relative sensitivity versus wavelength for the prior PCF with $\alpha=1.6 \mu \mathrm{m}$ and $d=1.4 \mu \mathrm{m}$.

for some distances between adjacent holes and the core diameter, respectively. As shown, by reducing the distance between the holes and also with increasing the core diameter, the relative sensitivity increases, because more evanescent field fraction spreads to the cladding and holes and interacts with gas samples. The sensitivity increases with an increase in the wavelength because the light can penetrate into the cladding holes by increasing the wavelength. The highest sensitivity obtained for $\alpha=1.6 \mu \mathrm{m}$ at the $\lambda=2.1 \mu \mathrm{m}$, is equal to $52 \%$. But according to the future simulations, the 
confinement loss is very high about $25 \mathrm{~dB} / \mathrm{m}$ in these conditions.

Figure 4 shows the calculated relative sensitivity for the proposed PCF with varying the distance between adjacent holes, $a$. With a reduction in $a$, from $2.3 \mu \mathrm{m}$ to $1.6 \mu \mathrm{m}$, the relative sensitivity increases because the cladding index reduces by a reduction in $a$, and so more light enters the cladding. Figure 5 shows the calculated confinement loss versus wavelength by changing the dimensions of the central hole. By decreasing the diameter of the central hole, the confinement loss will reduce because the difference of core and cladding indices is high, and consequently, more light power can be confined in the core region.

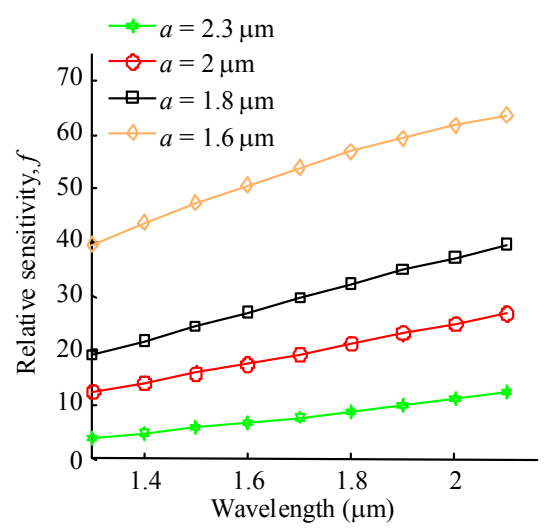

Fig. 4 Relative sensitivity versus wavelength for the proposed PCF with $d_{1}=1.4 \mu \mathrm{m}$ and $d_{c}=1 \mu \mathrm{m}$.

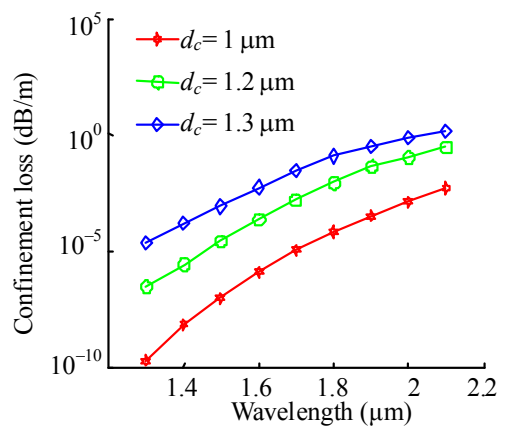

Fig. 5 Relative sensitivity versus wavelength for the proposed PCF with $\alpha=1.8 \mu \mathrm{m}$ and $d_{1}=1.4 \mu \mathrm{m}$.

Figure 6 compares the confinement loss curves for both the prior and proposed PCFs with the core diameter $d_{c}=1.2 \mu \mathrm{m}, 1 \mu \mathrm{m}$. For both PCFs, we considered $\alpha=1.6 \mu \mathrm{m}$ and $d_{1}=1.4 \mu \mathrm{m}$, and two outer ring holes dimension of the proposed PCF was $d_{2}=1.8 \mu \mathrm{m}$. Because the confinement loss difference between the two PCFs was too much, the logarithmic diagrams were plotted.

As it is clear from Fig. 6, the confinement loss increases with an increase in the core size. By increasing the core size, the index difference between the core and the cladding reduces, more light power can penetrate into the cladding region, and therefore, the confinement loss increases. The confinement loss at $\lambda=1.5 \mu \mathrm{m}$ for the proposed $\mathrm{PCF}$ is, whereas for the prior PCF, equal to $1.9 \mathrm{~dB} / \mathrm{m}$. According to Fig. 7, the relative sensitivities for the core diameter of $1 \mu \mathrm{m}$ are the same for both types of PCFs, and in the case of $1.2-\mu \mathrm{m}$ core diameter, $10 \%$ improved sensitivity for the proposed PCF is obtained.

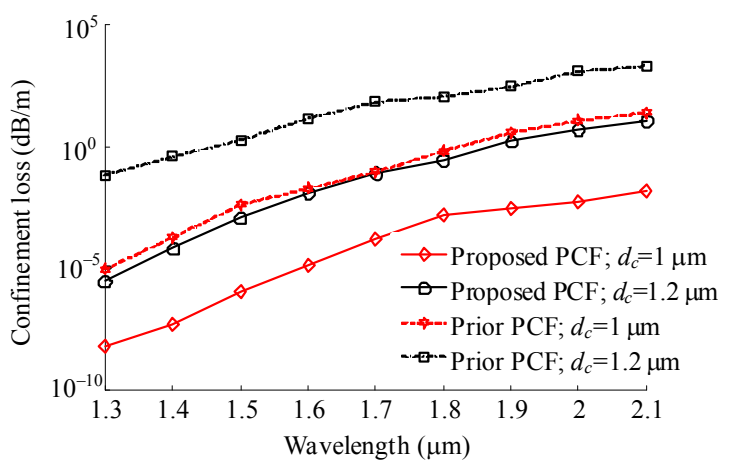

Fig. 6 Comparison of confinement losses in the prior PCF and the proposed PCF with $\alpha=1.6 \mu \mathrm{m}$ and $d_{1}=1.4 \mu \mathrm{m}$ and two core dimensions: $d_{\mathrm{c}}=1.2 \mu \mathrm{m}$ and $1 \mu \mathrm{m}$.

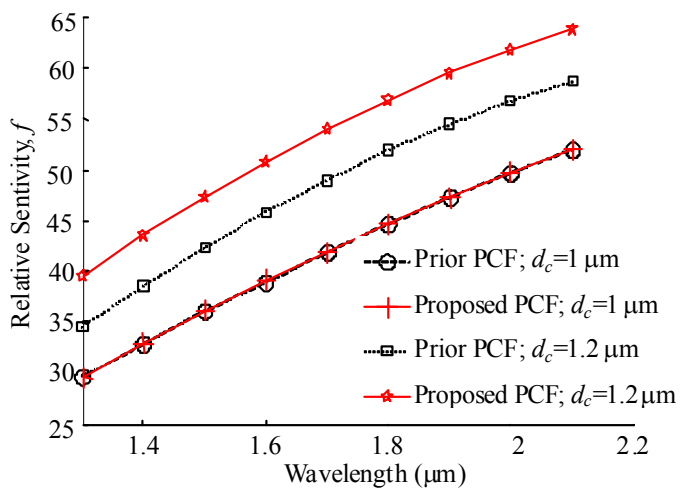

Fig. 7 Comparison of the relative sensitivity in the prior PCF and the proposed PCF with $\alpha=1.6 \mu \mathrm{m}$ and $d_{1}=1.4 \mu \mathrm{m}$ and two core dimensions: $d_{c}=1.2 \mu \mathrm{m}, 1 \mu \mathrm{m}$. 


\section{Conclusions}

A gas sensor based on the index guiding photonic crystal fiber is introduced. The dependency of sensor properties such as the relative sensitivity and confinement losses on the fiber structural parameters and working wavelength has been investigated. In the proposed fiber with optimized parameters at the wavelength of $1.5 \mu \mathrm{m}$, the relative sensitivity was improved to about 10 percent compared to the previous design. Also, the confinement loss for our design was about $6 \times 10^{-4}$ times less than that of the reference fiber reported in [18]. This result proved that the proposed PCF had a high capacity in the chemical and gas sensor applications.

Open Access This article is distributed under the terms of the Creative Commons Attribution License which permits any use, distribution, and reproduction in any medium, provided the original author(s) and source are credited.

\section{References}

[1] S. Olyaee and A. A. Dehghani, "High resolution and wide dynamic range pressure sensor based on two-dimensional photonic crystal," Photonic Sensors, vol. 2, no. 1, pp. 92-96, 2012.

[2] S. Olyaee and A. A. Dehghani, "Nano-pressure sensor using high quality photonic crystal cavity resonator," in 2012 8th International Symposium on Communication Systems, Networks \& Digital Signal Processing (CSNDSP), Poznan, July 18-20, pp. 1-4, 2012.

[3] J. C. Knight, T. A. Birks, P. St. J. Russell, and D. M. Atkin, "All-silica single-mode fiber with photonic crystal cladding," Optics Letters, vol. 21, no. 9, pp. 1547-1549, 1996.

[4] P. St. J. Russell, "Photonic crystal fibers," Science, vol. 299, no. 5605, pp. 358-362, 2003.

[5]M. Nielsen, C. Jacobsen, N. Mortensen, J. Folkenberg, and H. Simonsen, "Low-loss photonic crystal fibers for transmission systems and their dispersion properties," Optics Express, vol. 12, no. 7, pp. 1372-1376, 2004.

[6] S. Olyaee and F. Taghipour, "Doped-core octagonal photonic crystal fiber (O-PCF) with ultra-flattened nearly zero dispersion and low confinement loss in a wide wavelength range," Fibers and Integrated Optics, vol. 31, no. 3, pp. 178-185, 2012.

[7] W. N. MacPherson, E. J. Rigg, J. D. C. Jones, V. V. R. K. Kumar, J. C. Knight, and P. St. J. Russell, "Finite-element analysis and experimental results for a microstructured fiber with enhanced hydrostatic pressure sensitivity," Journal of Lightwave Technology, vol. 23, no. 3, pp. 1227-1231, 2005.

[8] B. Temelkuran, S. D. Hart, G. Benoit, J. D. Joannopoulos, and Y. Fink, "Wavelength-scalable hollow optical fibers with large photonic band gaps for $\mathrm{CO}_{2}$ laser transmission," Nature, vol. 420, no. 6916, pp. 650-653, 2002.

[9] S. Olyaee and F. Taghipour, "Ultra-flattened dispersion hexagonal photonic crystal fiber with low confinement loss and large effective area," IET Optoelectronics, vol. 6, no. 2, pp. 82-87, 2012.

[10] S. Olyaee and F. Taghipour, "A new design of photonic crystal fiber with ultra-flattened dispersion to simultaneously minimize the dispersion and confinement loss," Journal of Physics: Conference Series, vol. 276, no. 1, pp. 012080-1-012080-5, 2011.

[11] H. Du, "SERS-based photonic crystal fiber sensing platform," in Proc. SPIE, vol. 6083, pp. 74, 2006.

[12] J. C. Knight, "Photonic crystal fibres," Nature, vol. 424, no. 6950, pp. 847-851, 2003.

[13] S. Smolka, M. Barth, and O. Benson, "Highly efficient fluorescence sensing with hollow core photonic crystal fibers," Optics Express, vol. 15, no. 20, pp. 12783-12791, 2007.

[14] Y. L. Hoo, W. Jin, J. Ju, and H. L. Ho, "Numerical investigation of a depressed-index core photonic crystal fiber for gas sensing," Sensors and Actuators B: Chemical, vol. 139, no. 2, pp. 460-465, 2009.

[15] X. Yu, Y. C. Kwok, N. A. Khairudin, and P. Shum, "Absorption detection of cobalt (II) ions in an index-guiding microstructured optical fiber," Sensors and Actuators B: Chemical, vol. 137, no. 2, pp. 462-466, 2009.

[16] C. Martelli, J. Canning, D. Stocks, and M. J. Crossley, "Water-soluble porphyrin detection in a pure-silica photonic crystal fiber," Optics Letters, vol. 31, no. 14, pp. 2100-2102, 2006.

[17] J. Park, S. Lee, S. Kim, and K. Oh, "Enhancement of chemical sensing capability in a photonic crystal fiber with a hollow high index ring defect at the center," Optics Express, vol. 19, no. 3, pp. 1921-1929, 2011.

[18] Z. G. Zhang, F. D. Zhang, M. Zhang, and P. D. Ye, "Gas sensing properties of index-guided PCF with air-core," Optics \& Laser Technology, vol. 40, no. 1, pp. 167-174, 2008.

[19] K. Saitoh and M. Koshiba, "Full-vectorial 
imaginary-distance beam propagation method based on a finite element scheme: application to photonic crystal fibers," IEEE Journal of Quantum Electronics, vol. 38, no. 7, pp. 927-933, 2002.

[20] F. Brechet, J. Marcou, D. Pagnoux, and P. Roy, "Complete analysis of the characteristics of propagation into photonic crystal fibers, by the finite element method," Optical Fiber Technology, vol. 6, no. 2, pp. 181-191, 2000.

[21] S. Kunimasa and K. Masanori. "Leakage loss and group velocity dispersion in air-core photonic bandgap fibers," Optics Experss, vol. 11, no. 13, pp. 3100-3109, 2003.

[22] J. Wang, C. Jiang, W. Hu, and M. Gao, "Properties of index-guided PCF with air-core," Optics \& Laser Technology, vol. 39, no. 2, pp. 317-321, 2003.

[23] P. Viale, S. Fevrier, F. Gerome, and H. Vilard,
"Confinement loss computations in photonic crystal fibres using a novel perfectly matched layer design," presented at Proceedings of the COMSOL Multiphysics User's Conference, Paris, France, Nov. 15, 2005.

[24] K. Saitoh and M. Koshiba, "Confinement losses in air-guiding photonic bandgap fibers," IEEE Photonics Technology Letters, vol. 15, no. 2, pp. 236-238, 2003.

[25] D. Ferrarini, L. Vincetti, M. Zoboli, A. Cucinotta, and S. Selleri, "Leakage properties of photonic crystal fibers," Optics Express, vol. 10, no. 23, pp. 1314-1319, 2002.

[26] Y. L. Hoo, W. Jin, C. Shi, H. L. Ho, D. N. Wang, and S. C. Ruan, "Design and modeling of a photonic crystal fiber gas sensor," Applied Optics, vol. 42, no. 18, pp. 3509-3515, 2003. 